the rib to be anteriorly displaced and within $7 \mathrm{~mm}$ of the descending aorta (Figure 2). We elected to remove the rib fragment, and the patient was discharged within 1 week. Sata and colleagues ${ }^{6}$ also reported successful resection of a rib fragment seen to be migrating toward the thoracic aorta over serial CT scans. Sata and colleagues elected to perform open thoracotomy for rib resection, as in our case; however, a video-assisted thoracoscopic approach also may be a viable option in less complicated cases.

\section{CONCLUSIONS}

Although rib fracture-induced injury of the aorta is rare, given the severe implications of aortic injury, we recommend consideration of prophylactic resection of the rib fragments that threaten the aorta. Imaging studies such as
CT and 3-dimensional reconstructions may assist physicians in developing their threshold for prophylactic surgery in the future.

\section{References}

1. Sirmali M, Turut H, Topcu S, Gulhan E, Yazici U, Kaya S, et al. A comprehensive analysis of traumatic rib fractures: morbidity, mortality and management. Eur J Cardiothorac Surg. 2003;24:133-8.

2. Bruno V, Batchelor T. Late aortic injury: a rare complication of a posterior rib fracture. Ann Thorac Surg. 2009;87:301-3.

3. Marco J, Gregory J. Posterior fracture of the left sixth rib causing late aortic laceration: case report. J Trauma. 1997;42:736-7.

4. Akira I, Satoh N, Yamakawa H, Fujino M, Hiroshima K, Fujisawa T. Rupture of the descending thoracic aorta caused by blunt chest trauma: report of a case. Surg Today. 2003;33:755-7.

5. Ashrafian H, Kumar P, Sarkar P, DeSouza A. Delayed penetrating intrathoracic injury from multiple rib fractures. J Trauma. 2003;58:858-9.

6. Sata S, Yoshida J, Nishida T, Ueno Y. Sharp rib fragment threatening to lacerate the aorta in a patient with flail chest. Gen Thorac Cardiovasc Surg. 2007;55: $252-4$

\title{
Cardiac transapical approach: A new solution for the treatment of complex thoracic aortic disease
}

\author{
Bruno Borrello, MD, Francesco Nicolini, MD, PhD, Andrea Agostinelli, MD, PhD, and \\ Tiziano Gherli, MD, Parma, Italy
}

Endovascular repair of descending thoracic aorta pathology has become a well-recognized therapeutic option, ${ }^{1}$ although several patient results are unsuitable anatomically for standard treatment with current devices. ${ }^{2}$ Severe vascular occlusive disease may not allow the insertion of these large devices through the iliofemoral vessels or the abdominal aorta. We report a patient with impending rupture of a descending thoracic aorta aneurysm who underwent endovascular aortic repair using a transapical approach through the left ventricular apex.

\section{CLINICAL SUMMARY}

An 83-year-old man with history of systemic hypertension, dilated cardiomyopathy, chronic atrial fibrillation, diabetes mellitus, and chronic renal insufficiency was admitted to the emergency department of our hospital for

\footnotetext{
From the Heart Surgery Department, Parma University Hospital, Parma, Italy. Disclosures: Authors have nothing to disclose with regard to commercial support. Received for publication July 14, 2012; revisions received Aug 7, 2012; accepted for publication Aug 17, 2012; available ahead of print Sept 10, 2012.

Address for reprints: Francesco Nicolini, MD, PhD, Heart Surgery Section, Parma University Hospital, Via A. Gramsci 14, 43100 Parma, Italy (E-mail: francesco. nicolini@unipr.it).

J Thorac Cardiovasc Surg 2012;144:e123-5

$0022-5223 / \$ 36.00$

Copyright (C) 2012 by The American Association for Thoracic Surgery

http://dx.doi.org/10.1016/j.jtcvs.2012.08.039
}

cough with hemoptysis. A thoracoabdominal computed tomographic scan showed a multiple saccular pseudoaneurysm $(38 \times 57 \times 40 \mathrm{~mm})$ located on the left superolateral margin of the aortic wall, just beyond the origin of the left subclavian artery, with parietal thrombosis and perivascular hematoma (Figure 1, A). The lesion was complicated by the presence of 2 penetrating ulcers with bulging of the aortic wall. In this location, the inner layer of the aorta seemed to be very thin and partially interrupted. Angiography performed in the operating room confirmed the diagnosis. Unfortunately, emergency standard retrograde delivery of a thoracic stent graft was not feasible because of severe occlusive disease of the iliofemoral vessels (Figure 1,B). Moreover, the presence of a significant right subclavian artery tortuosity was judged to be an important and additional concern. Thus, considering the concomitant systemic comorbidities of the patient, and our experience with transapical aortic valve replacement, we decided that this minimally invasive approach would be the best option to deploy the endovascular aortic stent.

The patient was placed under general anesthesia and intubated with a single-lumen endotracheal tube. Imaging was provided by an angiographic C-arm system (Philips Veradius R1; Philips Healthcare, Best, The Netherlands). A left minithoracotomy incision was made in the fifth 

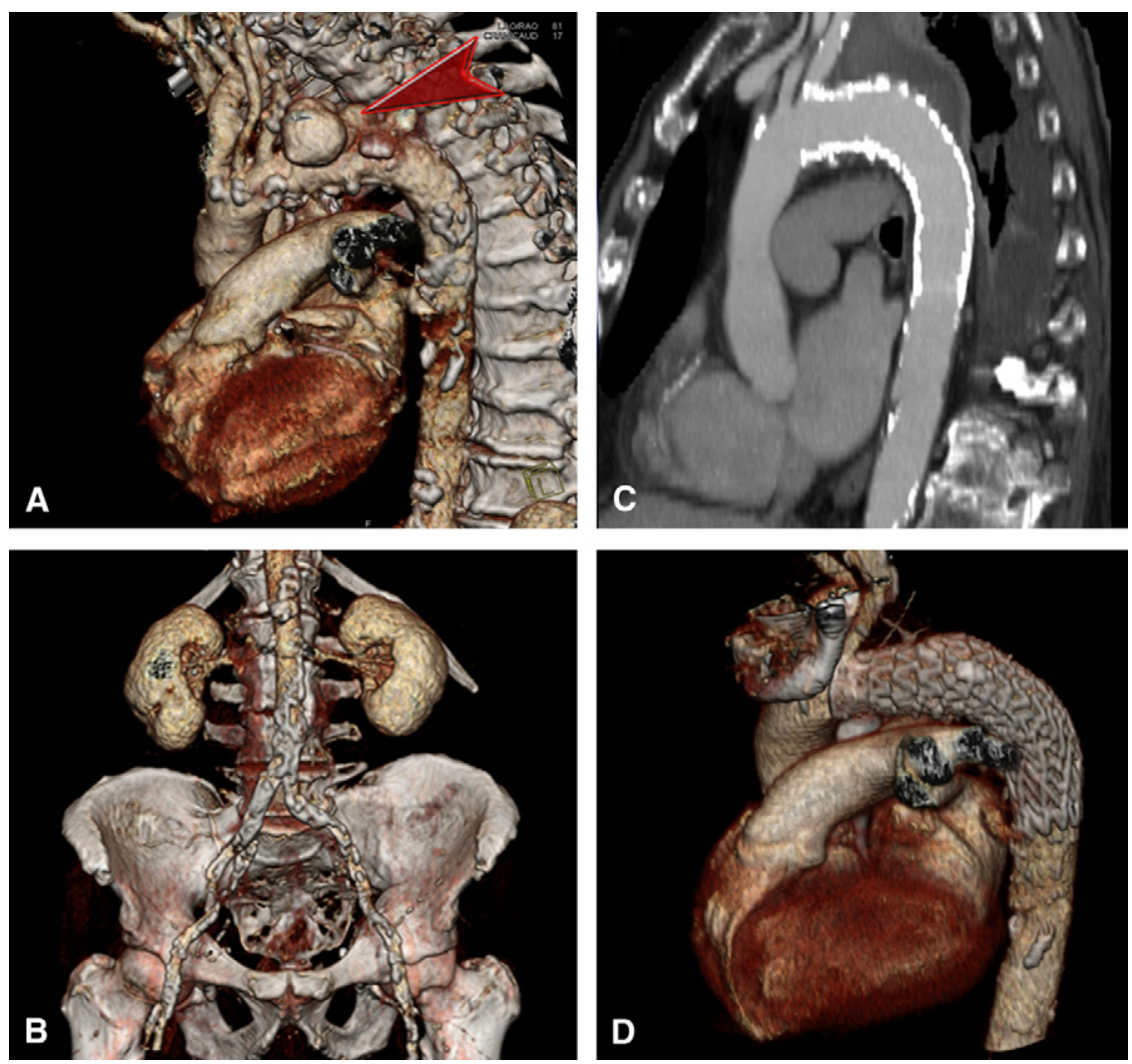

FIGURE 1. A, Preoperative left anterior oblique view of the thoracic aorta (volume rendering image). The arrowhead indicates the profile of the biggest saccular pseudo-aneurysm. B, Preoperative volume rendering image showing heavy calcification of the abdominal aorta and iliac branches. C and D, Postoperative multislice computed tomographic angiography at 2 weeks showing that the metallic grid is adhered to the aortic wall and the aneurismal sac is excluded correctly.

intercostal space. Using a rib retractor, the left ventricular apex was exposed and 2 pledgeted purse-string sutures were placed on it. The apex was accessed and a flexible guidewire was advanced across the aortic valve and into the descending aorta. After the insertion of a $22 \mathrm{~F}$ Gore DrySeal sheath (W. L. Gore \& Associates, Inc, Flagstaff, Ariz), the Gore Conformable TAG thoracic endoprosthesis $(15 \times 31 \mathrm{~mm}$; W. L. Gore \& Associates $)$ was prepared in the standard fashion and was advanced across the aortic valve and ascending aorta up to the descending thoracic aorta (Figure 2, A-C). Correct placement of the endoprosthesis was obtained at angiography by marking the origin of the left subclavian artery from the aortic arch with a guidewire positioned in the subclavian artery (Figure 2, C). Then, precise deployment was safely performed in the standard fashion, avoiding accidental coverage of the left subclavian artery (Figure 2,C). Aortic angiography demonstrated satisfactory endovascular repair, with the exclusion of the aneurysmal lesion and without any evidence of endoleak (Figure 2,D). Control computed tomographic scan performed 2 weeks after surgery confirmed the good results of the procedure (Figure 1, $C$ and $D$ ).

\section{DISCUSSION}

Antegrade strategies for the placement of aortic stent grafts such as the axillary approach, ascending aortic delivery, or direct placement of aortic stent grafts have been investigated. ${ }^{3}$ These techniques have proved to be effective in the treatment of complex thoracic aortic disease that is not suitable anatomically or physiologically for the usual approaches.

To the best of our knowledge, this is the first report of a mini-invasive transapical delivery of a stent graft for the repair of a complex descending thoracic aorta aneurysm. Technical success obtained in this case demonstrates that endovascular treatment of thoracic aortic disease can be performed safely in select patients by a left minithoracotomy and a transapical ventricular approach. This technique could be particularly useful in patients in whom standard retrograde delivery of thoracic stent graft results is not feasible because of severe occlusive disease of the iliofemoral vessels, because antegrade deployment of an aortic endoprosthesis is technically demanding as a result of previous cardiac surgery, or because of an anatomic tortuosity of the right subclavian artery. Although our strategy proved to be safe and effective during the early period, the 


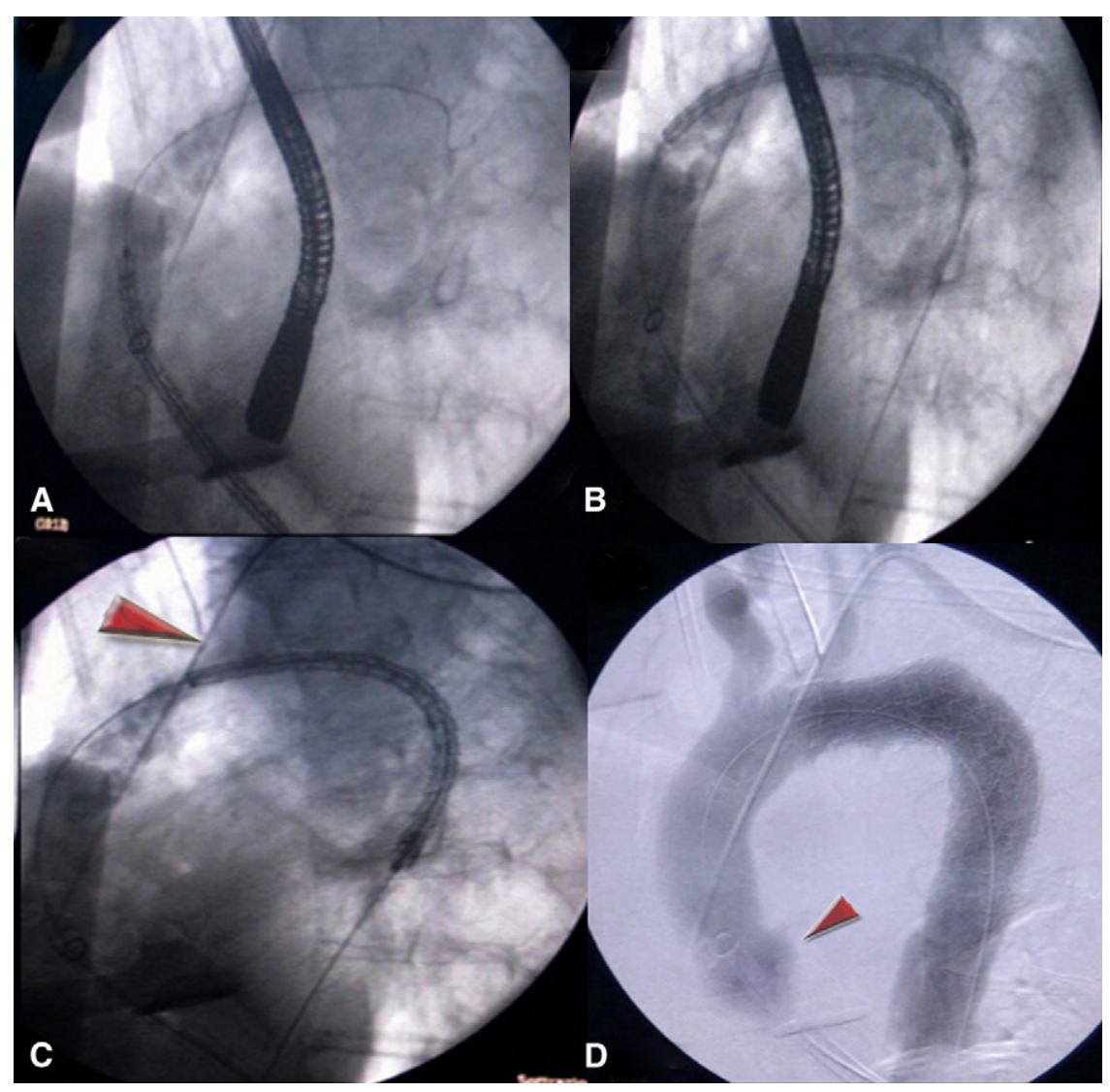

FIGURE 2. Left anterior oblique angiogram during the endovascular procedure. A, The guidewire is in the aortic arch and the endoprosthesis is proceeding along the ascending aorta. B, the endoprosthesis is pushed forward in the aortic arch. $\mathrm{C}$, The endoprosthesis reaches the correct position when it crosses the guidewire in the subclavian artery (landmark indicated by the arrowhead). D, Angiogram immediately after endoprosthesis opening showing the restoration of normal aortic caliber without contrast leakage (arrowhead indicates the aortic root).

long-term outcome of these new strategies needs to be evaluated further.

\section{References}

1. Gopaldas RR, Huh J, Dao TK, LeMaire SA, Chu D, Bakaeen FG, et al. Superior nationwide outcomes of endovascular versus open repair for isolated descending thoracic aortic aneurysm in 11,669 patients. J Thorac Cardiovasc Surg. 2010; 140:1001-10

2. Roselli EE, Greenberg RK, Pfaff K, Francis C, Svensson LG, Lytle BW. Endovascular treatment of thoracoabdominal aortic aneurysms. J Thorac Cardiovasc Surg. 2007; $133: 1474-82$.

3. Roselli EE, Soltesz EG, Mastracci T, Svensson LG, Lytle BW. Antegrade delivery of stent grafts to treat complex thoracic aortic disease. Ann Thorac Surg. 2010;90: $539-46$. 